\section{A gut feeling about butyrate}

\section{By Tracey Baas, Senior Editor}

Inflammatory bowel disease and other inflammatory conditions of the gut have long been associated with imbalances in mucosal levels of microflora-produced short-chain fatty acids such as butyrate and propionate, but the underlying mechanisms were unknown. Work from two separate groups in the U.S. and Japan has now revealed how bacterial butyrate and propionate can drive the differentiation of antiinflammatory $\mathrm{T}_{\mathrm{reg}}$ cells and thus promote mucosal health. ${ }^{1,2}$

Both teams are trying to find links between their findings and other immune-related conditions, such as autoimmune diseases and food allergies, while also looking into suitable therapeutic modalities for gut inflammatory conditions.

Butyrate insufficiency caused by low levels of butyrateproducing bacteria or downregulation of butyrate transporters in the colonic mucosa of patients with IBD has been implicated in IBD pathogenesis. ${ }^{3,4}$

In patients with IBD, enema treatment with butyrate or with a cocktail of short-chain fatty acids (SCFAs) ameliorates colonic inflammation via an unknown mode of action. ${ }^{5,6}$

A Japan team led by Hiroshi Ohno and a U.S. team led by Alexander Rudensky have independently elucidated one underlying mechanism.

Ohno is group director of the Laboratory for Epithelial Immunobiology at the RIKEN Center for Integrative Medical Sciences, and Rudensky is the immunology program chair and director of the Ludwig Center for Cancer Immunotherapy at the Memorial Sloan-Kettering Cancer Center.

The Japan team included researchers from The University of Tokyo, Keio University and the Commonwealth Scientific and Industrial Research Organisation Food and Nutritional Sciences.

Based on previous studies showing that $\mathrm{T}_{\text {reg }}$ cells expressing forkhead box P3 (FOXP3) play a key role in limiting inflammatory responses in the intestine, ${ }^{7}$ both teams first set out to determine whether butyrate or other SCFAs would influence $\mathrm{T}_{\text {reg }}$ cell differentiation ex vivo. Under $\mathrm{T}_{\text {reg }}$ cell-inducing conditions, naïve mouse $\mathrm{Cd} 4^{+} \mathrm{T}$ cells exposed to butyrate or fecal extracts obtained from mice with commensal bacteria exhibited greater $\mathrm{T}_{\text {reg }}$ cell differentiation and higher numbers of Foxp $3^{+}$ $\mathrm{T}_{\text {reg }}$ cells than Cd4 $4^{+} \mathrm{T}$ cells exposed to other SCFAs or fecal extracts obtained from mice treated with antibiotics.

In the mice with commensal bacteria, the fecal extracts contained high levels of butyrate and propionate.

Next, both teams showed that in mice with normal gut flora, a diet containing butyrylated starch increased levels of colonic $\mathrm{T}_{\text {reg }}$ cells compared with a diet containing acetylated or propionylated starch, indicating that butyrate plays a key role in microbe-mediated $\mathrm{T}_{\text {reg }}$ cell induction. Importantly, butyrylated starch had no effect on antibiotictreated or germ-free mice, suggesting that commensal bacteria are required for $\mathrm{T}_{\text {reg }}$ cell induction.

Previous studies have established that butyrate can regulate gene expression epigenetically by inhibiting histone deacetylases (HDACs), ${ }^{8,9}$ and class IIa HDAC has been further reported to suppress $\mathrm{T}_{\text {reg }}$ cell expansion. ${ }^{10,11}$

Both teams thus went on to show that under $\mathrm{T}_{\text {reg }}$ cell-inducing conditions, butyrate led to increased histone $\mathrm{H} 3$ acetylation at Foxp3 promoter and enhancer elements and correlated with increased Foxp3 expression and $\mathrm{T}_{\text {reg }}$ cell differentiation compared with no butyrate. These results established that butyrate contributes to $\mathrm{T}_{\text {reg }}$ cell

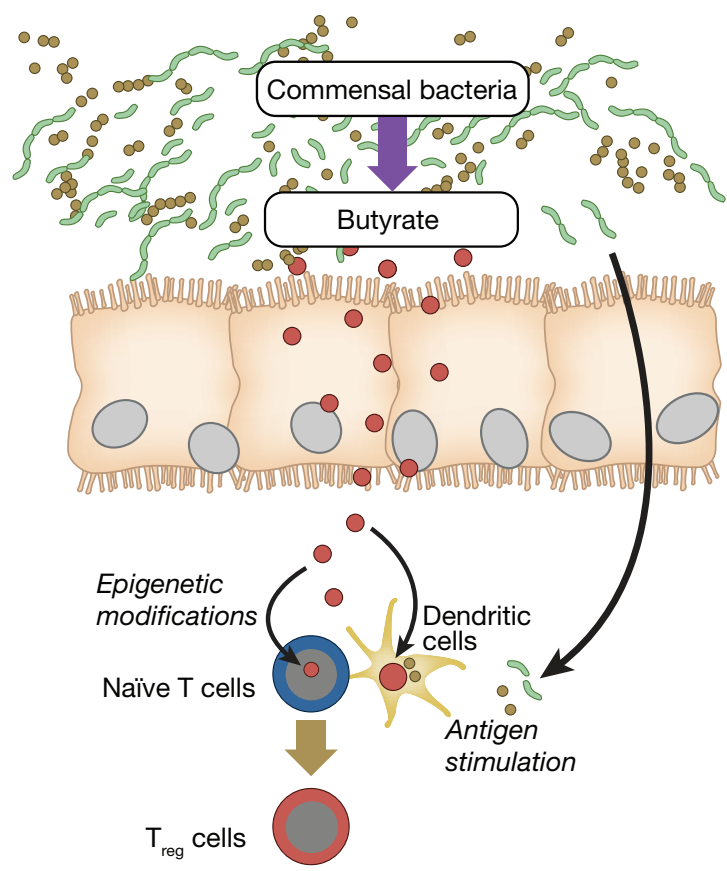

Figure 1. The commensal bacteria-butyrate- $T_{\text {reg }}$ cell axis of inflammatory regulation in the gut. Certain commensal bacteria abundantly produce butyrate through fermentation of dietary fiber and other substrates. Two recent studies ${ }^{1,2}$ found that butyrate promotes histone $\mathrm{H} 3$ lysine acetylation of the promoter and enhancer elements of forkhead box P3 (Foxp3) in naïve mouse $\mathrm{Cd} 4{ }^{+}$ $T$ cells, eventually inducing the differentiation of $T_{\text {reg }}$ cells. $T_{\text {reg }}$ cells in turn contribute to mucosal health by regulating the inflammatory response in the gut.

Ex vivo, Arpaia et al. showed that greater levels of Foxp3 acetylation and $T_{\text {reg }}$ cell differentiation could be achieved by coculturing butyrate-treated dendritic cells with naïve $\mathrm{Cd} 4^{+} \mathrm{T}$ cells rather than directly treating naïve $\mathrm{Cd} 4^{+} \mathrm{T}$ cells with butyrate. These results suggest that butyrate works through more than one immune cell population. (Figure based on Supplemental Figure 22 in ref. 1.) 
differentiation epigenetically (see Figure 1, "The commensal bacteriabutyrate- $\mathrm{T}_{\text {reg }}$ cell axis of inflammatory regulation in the gut”).

Finally, the Japan researchers showed that a diet containing butyrylated starch prevented colitis in a mouse model of chronic intestinal inflammation. Diets containing normal starch did not prevent disease development. These disease-modifying results were seen in mice with normal levels of $\mathrm{T}_{\text {reg }}$ cells but not in mice without $\mathrm{T}_{\text {reg }}$ cells, indicating butyrate exerted its therapeutic activity through $\mathrm{T}_{\text {reg }}$ cells.

Results from both teams were published in Nature.

"Thus far the precise mechanisms of how bacteria communicate with their hosts to promote immune functions have been poorly understood," said Carolin Daniel. "The present studies add to our understanding that microbes that metabolize dietary fiber can generate key fatty acids that enforce the induction of regulatory T cells."

Daniel is junior group leader of immune tolerance in type 1 diabetes at the Institute of Diabetes Research at Helmholtz Center Munich.

"While our work may raise considerable interest in butyrate, elucidation of the modes of action and range of effects demand further investigation," said Julie Clarke, an author on the Japan team's manuscript and project leader for the Starplus Starch Technology in CSIRO's Preventative Health Flagship. "More animal studies are required before any human disease intervention studies can be considered."

Indeed, Diane Mathis told SciBX, "Only one colitis model has been studied and, being a quite artificial transfer model, I'd like to see the effect on better, especially spontaneous, disease models." Mathis is a professor of microbiology and immunology at Harvard Medical School.

"I'd also like to see that these SCFAs can expand human $\mathrm{T}_{\text {reg }}$ cells and under what conditions," Mathis said. It would be helpful to characterize "what the SCFA landscape is like in humans, both in normal and diseased individuals."

Previous studies from CSIRO and collaborators showed that butyrylated starch is effective at delivering significant quantities of butyrate to the large bowel of humans, ${ }^{12}$ but those studies also showed that butyrylated starch had limited effects on humoral immunity in healthy individuals. ${ }^{13}$

Daniel noted that the CSIRO studies call into question whether the application of butyrylated starch would be the approach of choice.

"One potential approach would be to feed specific substratesprebiotics - that are suited to preferentially expand beneficial bacteria that can produce butyrate," said Daniel. "Alternatively, one could think of isolating beneficial bacterial species and enrich them within the existing bacterial community of the host."

\section{Further butyrate potential}

Besides the prospects of harnessing butyrate as a therapeutic for IBD, the results open opportunities in a range of other inflammatory, $\mathrm{T}_{\text {reg }}$ cell-dependent diseases.

"It would be interesting to see if the findings apply not only to mucosal inflammation in the intestine but also to organ-specific autoimmune diseases such as type 1 diabetes and sterile inflammation," said Shimon Sakaguchi, a professor of experimental immunology at the Immunology Frontier Research Center at Osaka University.
Mathis was less sanguine, noting, "There is more and more evidence that $\mathrm{T}_{\text {reg }}$ cells come in multiple flavors and that inflamed tissues have their own unique populations controlling autoimmunity and inflammatory responses. It is not yet clear to what extent the $\mathrm{T}_{\text {reg }}$ cells generated in the gut or systemically under these conditions will be able to perform all surveillance and homeostatic functions. Butyrateinduced $\mathrm{T}_{\text {reg }}$ cell generation might work for some diseases, especially in the intestine, but not others."

But Sakaguchi added, "Metabolites as well as environmental cues produced by commensal bacteria contain a wide range of immune modulators for innate and adaptive immune cells, so further detailed analyses will be needed to understand integrated immune responses and develop a measure to control the immune balance."

"Regulatory T cells do play an essential role to avoid excessive inflammatory responses as well as autoimmunity. Therefore, the beneficial effects of butyrate could be applicable for the prevention and possibly also treatment of other autoimmune diseases or allergies-including food allergies-where $\mathrm{T}_{\text {reg }}$ cells have been implied to be of relevance," said Daniel. "With respect to inflammatory bowel disease it would be helpful to see specific outcomes in models for Crohn's disease vs. ulcerative colitiswhether butyrate is equally beneficial in both diseases."

Ohno's team is planning to explore the role of butyrate
"It would be interesting to see if the findings apply not only to mucosal inflammation in the intestine but also to organspecific autoimmune diseases such as type 1 diabetes and sterile inflammation."

-Shimon Sakaguchi,

Immunology Frontier Research Center at Osaka University allergy and to see if they can influence the experimental autoimmune encephalomyelitis (EAE) mouse model of multiple sclerosis (MS).

The team is also investigating alternative delivery methods to starch, but Ohno declined to give further details.

Rudensky's team is now investigating how butyrate and colonic $\mathrm{T}_{\text {reg }}$ cells play a role in autoimmune models of diabetes, colitis and rheumatoid arthritis (RA). The team is also looking into therapeutic modalities to bypass use of starch, but Rudensky declined to give further details.

The Japan team has filed a patent application covering the use of butyrate to induce differentiation of colonic $\mathrm{T}_{\text {reg }}$ cells as a potential therapeutic for inflammatory, autoimmune and allergic diseases. The IP is unlicensed.

Acylation of starches and other fiber polysaccharides for the delivery of SCFAs to the large bowel is patented by CSIRO and is available for licensing.

The U.S. team has filed a provisional patent application protecting methods and compositions for modulating inflammation and treating $\mathrm{T}_{\text {reg }}$ cell-associated diseases. The IP is available for licensing from the Office of Technology Development at the Memorial Sloan-Kettering Cancer Center.

Baas, T. SciBX 6(46); doi:10.1038/scibx.2013.1310

Published online Dec. 5, 2013 


\section{ANALYSIS}

\section{TARGETS \& MECHANISMS}

\section{REFERENCES}

1. Furusawa, Y. et al. Nature; published online Nov. 13, 2013; doi:10.1038/nature12721

Contact: Hiroshi Ohno, RIKEN Center for Integrative Medical

Sciences, Kanagawa, Japan

e-mail: ohno@rcai.riken.jp

Contact: Koji Hase, same affiliation as above

e-mail: hase@ims.u-tokyo.ac.jp

Contact: Shinji Fukuda, same affiliation as above

e-mail: sfukuda@sfc.keio.ac.jp

2. Arpaia, N. et al. Nature; published online Nov. 13, 2013; doi:10.1038/nature12726

Contact: Alexander Y. Rudensky, Memorial Sloan-Kettering Cancer Center, New York, N.Y.

e-mail: rudenska@mskcc.org

3. Thibault, R. et al. Gastroenterology 133, 1916-1927 (2007)

4. Frank, D.N. et al. Proc. Natl. Acad. Sci. USA 104, 13780-13785 (2007)

5. Scheppach, W. et al. Gastroenterology 103, 51-56 (1992)

6. Harig, J.M. et al. N. Engl. J. Med. 320, 23-28 (1989)

7. Josefowicz, S.Z. et al. Nature 482, 395-399 (2012)
8. Candido, E.P.M. et al. Cell 14, 105-113 (1978)

9. Davie, J.R. J. Nutr. 133, 2485S-2493S (2003)

10. de Zoeten, E.F. et al. Gastroenterology 138, 583-594 (2010)

11. Tao, R. et al. Nat. Med. 13, 1299-1307 (2007)

12. Clarke, J.M. et al. Am. J. Clin. Nutr. 94, 1276-1283 (2011)

13. West, N.P. et al. Exerc. Immunol. Rev. 19, 102-119 (2013)

\section{COMPANIES AND INSTITUTIONS MENTIONED}

Commonwealth Scientific and Industrial Research Organisation Food and Nutritional Sciences, Adelaide, South Australia, Australia Harvard Medical School, Boston, Mass.

Immunology Frontier Research Center at Osaka University,

Osaka, Japan

Institute of Diabetes Research at Helmholtz Center Munich,

Munich, Germany

Keio University, Yamagata, Japan

Ludwig Center for Cancer Immunotherapy at the Memorial

Sloan-Kettering Cancer Center, New York, N.Y.

RIKEN Center for Integrative Medical Sciences, Kanagawa, Japan

The University of Tokyo, Tokyo, Japan 